\title{
System Modeling and Characterization of SiC Schottky Power Diodes
}

\author{
Hui Zhang, Student Member, IEEE, Leon M. Tolbert, Senior Member, IEEE, Burak Ozpineci, Senior Member, IEEE
}

\begin{abstract}
Most of the present models of silicon carbide (SiC) Schottky diodes are not suitable for evaluating their performance from a system level. The models presented in this paper are specialized for system-level simulations. They are based on basic semiconductor theories and synthesis of some models in the literature. Theoretical and experimental characterization of SiC Schottky power diodes is also involved. The models describe both static and dynamic behaviors of $\mathrm{SiC}$ Schottky power diodes. Thermal effects are considered as well for a better evaluation of power losses evaluation and cooling system design. The models were also used to estimate the efficiencies of Si IGBT/SiC Schottky diode hybrid inverter. To validate the simulation, a Si IGBT/SiC Schottky diode hybrid inverter and a Si IGBT inverter were built and tested.
\end{abstract}

Keywords - Silicon Carbide (SiC), Schottky diode, modeling, temperature, hybrid inverter

\section{INTRODUCTION}

The increasing needs for higher-voltage, more efficient, and more reliable power electronic devices have given birth to the rapid progress of silicon carbide ( $\mathrm{SiC}$ ) semiconductor technology. The advantages of $\mathrm{SiC}$ power devices have been recognized by both the academia and industry. At present, SiC Schottky power diodes, as the first commercially available $\mathrm{SiC}$ power devices, are ready to replace their $\mathrm{Si}$ counterparts in many applications in order to obtain higher efficiency, less cooling requirement, or higher compactness of systems.

Efficient device models are required to evaluate the performance of SiC Schottky diodes in different applications and guide system design. Although several models have been developed for SiC Schottky diodes, most of them are based on device physics or based on experiments [1] [2]. For the former, usually a number of device parameters (which are usually known only by designers) are required to solve the model, and sometimes the model itself is complicated, and difficult to solve or time consuming. For the latter, a variety of experiments are needed. Parameter extractions are also involved and can be rather tough. Accordingly, these models are difficult to be integrated into a system simulation. It is necessary to find some models of SiC Schottky power diodes specialized for system modeling. This work is to address this need.

\section{Modeling of SiC Schottky Power Diodes}

In this section, SiC Schottky diodes are characterized by both theoretical analysis and experiments. The models for static state (forward conduction) and dynamic state (reverse recovery) will be discussed respectively.

\section{A. Static State}
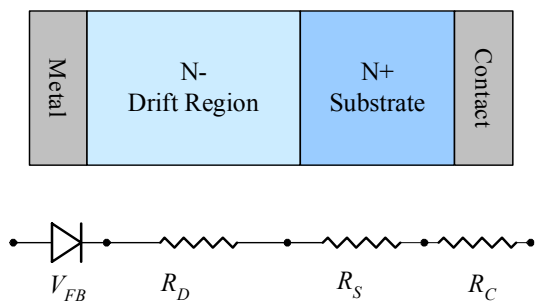

Fig. 1. Basic structure and equivalent circuit of Schottky power diodes.

The structure of a SiC Schottky power diode and its equivalent circuit are shown in Fig. 1. $V_{F B}$ is the voltage drop across the Schottky barrier; $R_{D}$ is the resistive voltage drop across the lightly doped drift region; $R_{S}$ and $R_{C}$ are the resistance of substrate and contact, respectively.

In a $\mathrm{SiC}$ Schottky power diode, the thermionic emission process dominates in the transport of current across a metal $n$-type semiconductor contact. Under the forward bias condition, the current flow across the Schottky barrier is given by [3]

$$
J_{F}=C T^{2} e^{q\left(V_{F B}-\phi_{B}\right) / k T},
$$

where $\phi_{B}$ is the barrier height between the metal and $n$-type semiconductor, $T$ is the absolute temperature, $q$ is the charge of an electron, $k$ is Boltzmann's constant, $C$ is Richardson's constant, which is given by

$$
C=\frac{4 \pi m k^{2} q}{h^{3}},
$$

where $m$ is the effective mass of an electron, and $h$ is Plank's constant. For $4 \mathrm{H}-\mathrm{SiC}$, the theoretical value of Richardson's constant is $146 \mathrm{~A} \cdot \mathrm{cm}^{-2} \cdot \mathrm{K}^{-2}[4]$.

Solve (1) for $V_{F B}$, and neglect $R_{S}$ and $R_{C}$ (because they are usually small compared to $R_{D}$ for power devices with breakdown voltage larger than $200 \mathrm{~V}$ ), then the total voltage drop across a Schottky power diode can be expressed as

$$
V_{F}=\phi_{B}+\frac{k T}{q} \ln \left(\frac{J_{F}}{C T^{2}}\right)+J_{F} R_{D},
$$

where $R_{D}$ is given by

$$
R_{D}=\frac{4 V_{B}^{2}}{\varepsilon E_{c}^{3} \mu_{n}},
$$


$V_{B}$ is the breakdown voltage; $E_{c}$ is the breakdown electrical field; $\mu_{n}$ is electron mobility, which is a function of doping density $N_{d}$, electrical field $E$, and temperature $T$ in the depletion layer. As paper [5], $\mu_{n}$ can be expressed as

$$
\begin{aligned}
& \mu_{n}=\frac{\mu_{0}}{\left[1+\left(\frac{\mu_{0} E}{v_{s}}\right)^{\beta}\right]^{1 / \beta}}, \\
& \mu_{0}=\mu_{\min }+\frac{\mu_{\max }-\mu_{\min }}{1+\left(N_{\text {tot }} / N_{\text {ref }}\right)^{\alpha}}, \\
& v_{s}(T)=\frac{v_{\max , 600 K}}{1+0.8 \cdot \exp \left(\frac{T}{600}\right)}, \\
& \mu_{\max }=A_{\mu_{\max }} \times\left(\frac{T}{300}\right)^{-B_{\mu_{\max }}}, \\
& \mu_{\min }=A_{\mu_{\min }} \times\left(\frac{T}{300}\right)^{-B_{\mu_{\min }}} \text {, } \\
& N_{r e f}=A_{N_{r e f}} \times\left(\frac{T}{300}\right) .
\end{aligned}
$$

Eqs. (3)-(10) give a comprehensive description of the static characteristics of SiC Schottky power diodes. The coefficients involved can be found in many papers (like [6$8]$ ), and only the breakdown voltage of devices is needed to solve the equations. Using this model to estimate the forward characteristic of a $1200 \mathrm{~V} / 10 \mathrm{~A} 4 \mathrm{H}-\mathrm{SiC}$ Schottky power diode, a good match between the test and the calculation results is obtained as shown as Fig. 2. The parameters used in the calculation are listed in Table I.

Differentiation of Eq. (3) with respect to $J_{F}$ yields the onstate specific resistance of a Schottky power diode:

TABLE I. PARAMETERS USED IN SIMULATION

\begin{tabular}{|l|c|}
\hline \multicolumn{1}{|c|}{ Property } & $4 \mathrm{H}-\mathrm{SiC}$ \\
\hline Breakdown electric field, $E_{c}(\mathrm{kV} / \mathrm{cm})$ & 2200 \\
\hline Relative dielectric constant, $\varepsilon$ & 10.1 \\
\hline Doping coefficient of $\mu, \alpha$ & $0.76[7]$ \\
\hline Electric field coefficient of $\mu, \beta$ & $1[7]$ \\
\hline Coefficient of $\mu_{\max }, A_{\mu_{\max }}$ & $950[7[$ \\
\hline Coefficient of $\mu_{\max }, B_{\mu_{\max }}$ & $2.4[7]$ \\
\hline Coefficient of $\mu_{\min }, A_{\mu_{\min }}$ & $40[7]$ \\
\hline Coefficient of $\mu_{\min }, B_{\mu_{\min }}$ & $0.5[7]$ \\
\hline Coefficient of $N_{r e f}, A_{N_{r e f}}$ & $2 \times 10^{17}[7]$ \\
\hline Maximum saturated velocity, $v_{s \max }(\mathrm{cm} / \mathrm{s})$ & $4.77 \times 10^{7}[7]$ \\
\hline Schottky barrier height, $\phi_{B}(\mathrm{eV})$ & $1.25[9]$ \\
\hline Richardson's constant, $A\left(\mathrm{~A} \cdot \mathrm{cm}^{-2} \cdot \mathrm{K}^{-2}\right)$ & $146[4]$ \\
\hline
\end{tabular}

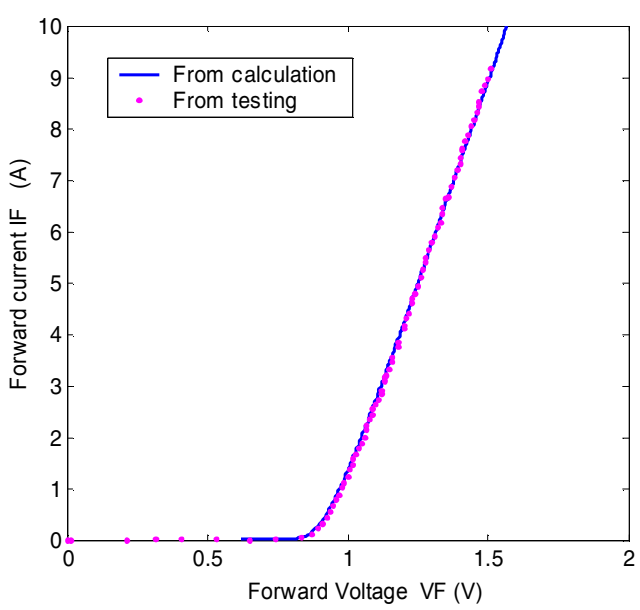

Fig. 2. Forward characteristic of SiC Schottky power diode.

$$
R_{s p}=\frac{d V_{F}}{d J_{F}}=\frac{k T}{q}\left(\frac{1}{J_{F}}\right)+R_{D} .
$$

It indicates the dependence of this on-state specific resistance on temperature and forward current. Moreover, as the forward current increases, the contribution of the first component in (11) becomes smaller and can be neglected. This means that the on-state resistance is nearly constant regardless of forward current and only changes with temperature at a relative high current region $(>1 \mathrm{~A}$ in this case), which corresponds to the linear region in Fig. 2. Most SiC Schottky power diodes operate in this region. Thus, it is reasonable to only consider drift region resistance $R_{D}$ in system modeling. Correspondingly, the temperature dependence of the on-state resistance is determined by the change of $\mu_{n}$ with temperature.

Fig. 3 compares simulation results of the on-state resistance of several commercially available SiC Schottky diodes to the experimental results (from datasheet [10-12]) at

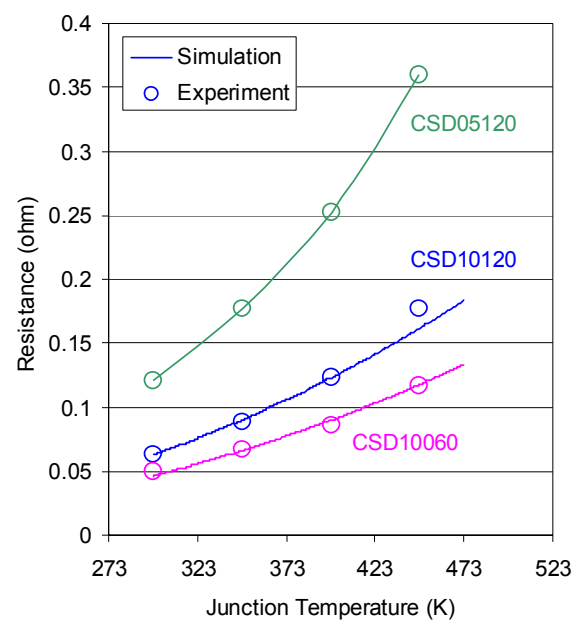

Fig. 3. On-state resistance of SiC Schottky diodes (Cree). 
different junction temperatures. Small errors were found, which indicates that these models are effective in predicting the temperature-dependent static characteristics of $\mathrm{SiC}$ Schottky diodes.

\section{B. Dynamic State}

Reverse recovery is the most important dynamic behavior of Schottky power diodes. Since there is no minority carrier injection in Schottky power diodes, the depletion layer capacitance determines their behaviors during reverse recovery. When a reverse biased voltage $V_{R}$ is applied to a Schottky power diode, from the well-known device theory [13], the width of its depletion layer can be calculated by

$$
w_{d}=\sqrt{\frac{2 \varepsilon\left(V_{R}+\phi_{B}\right)}{q N_{d}}} .
$$

Then, the specific depletion layer capacitance can be presented as

$$
C_{d}=\frac{\varepsilon}{w_{d}}=\sqrt{\frac{q N_{d} \varepsilon}{2\left(V_{R}+\phi_{B}\right)}} .
$$

As shown by (13), $C_{d}$ is a strong function of $V_{R}$, but is not affected by the current flowing through it. That is to say, the switching loss of a SiC Schottky diode mainly depends on the reverse voltage. Thus, in system modeling, it is reasonable to model the reverse recovery charge of $\mathrm{SiC}$ Schottky diodes as a function of their reverse voltage. Specifically, the reverse-recovery charge increases approximately linear with $V_{R}^{0.5}$, and the energy loss during this period increases linearly with $V_{R}^{1.5}$ (see (13)).

To further validate this argument, a SiC Schottky diode (Cree CSD10120) was tested at different reverse voltage with the same forward current. The turn-off characteristics are shown in Fig. 4. As expected, the reverse-recovery charge increased as the reverse voltage increased, and the ratio of reverse-recovery charge at $315 \mathrm{~V}$ to that at $150 \mathrm{~V}$ is 1.452 , which coincides with the square root of $315 \mathrm{~V} / 150 \mathrm{~V}$ (1.449). In addition, if the slight changes of $\varepsilon$ and $\phi_{B}$ with

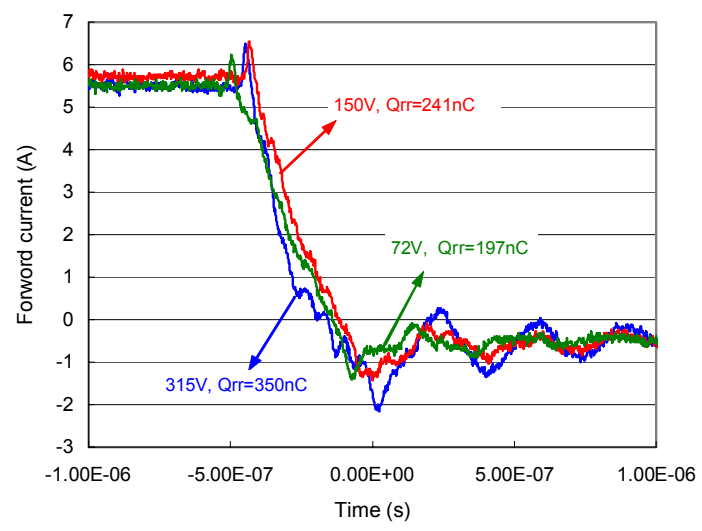

Fig. 4. Reverse recovery waveforms of the SiC Schottky diode. temperature are not considered, the reverse recovery behavior of SiC Schottky diodes will be the same at any temperature. This is also consistent with the test results in paper [14]. Thus, in system modeling, the influence of temperature on the reverse recovery characteristics of $\mathrm{SiC}$ Schottky diodes can be neglected.

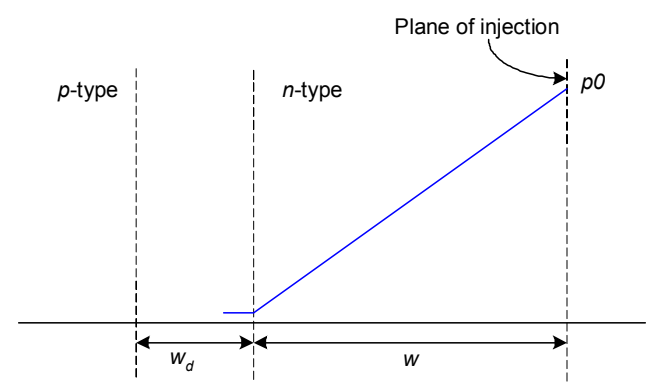

Fig. 5. Injected minority carrier (holes) density vs. position in reverse-biased $p-n$ junction.

Compared to SiC Schottky diodes, the switching loss of $\mathrm{Si}$ diodes during reverse recovery is much larger. This is because most of the Si diodes used in power applications have a $p$ - $n$ junction structure. For this type of diodes, besides the depletion layer capacitance, there is an additional capacitance due to the minority carrier injection under reverse biased condition. As shown in [15], the injected minority carrier density in a reverse-biased $p$ - $n$ junction decreases approximately linearly through the distance $w$ (shown in Fig. 5) and becomes negligible at the edge of the junction, the total charge and the capacitance due to minority carrier injection can be therefore expressed as

$$
\begin{gathered}
Q_{I}=\frac{J_{F} w}{2 D_{p}}, \\
C_{I}=-\frac{\partial Q_{I}}{\partial V_{R}}=\frac{J_{F} w}{D_{p}} \sqrt{\frac{\varepsilon\left(N_{d}+N_{a}\right)}{2 q N_{d} N_{a}\left(V_{R}+\phi_{R}\right)}} .
\end{gathered}
$$

where $N_{d}$ and $N_{a}$ are the doping density in n-type and p-type regions, respectively. $D_{P}$ is the diffusion constant of holes.

In accordance with the above two equations, the capacitance due to minority carrier injection dominates the reverse recovery characteristic, especially for high current level. As a result, for Si diodes, the total charge swept out during turn off is more dependent on the forward current than the reverse voltage. The test results reflect the same facts. As shown as Fig. 6, the reverse-recovery charge of the tested Si diode increased quickly as the forward current increased, while its variation with reverse voltage was much less (see Fig. 7). Therefore, for a system with current changing from time to time, the influence of the forward current on the reverse recovery of $\mathrm{Si}$ diodes must be considered. This is one of the important factors that account for the larger power loss of $\mathrm{Si}$ diodes compared to $\mathrm{SiC}$ Schottky diodes. 


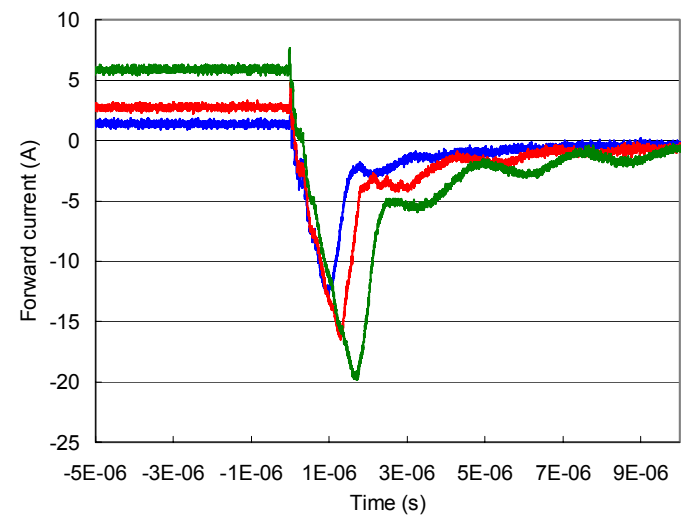

Fig. 6. Reverse recovery waveforms of the Si diode at $V_{R}=150 \mathrm{~V}$ (IR 20ETS08).

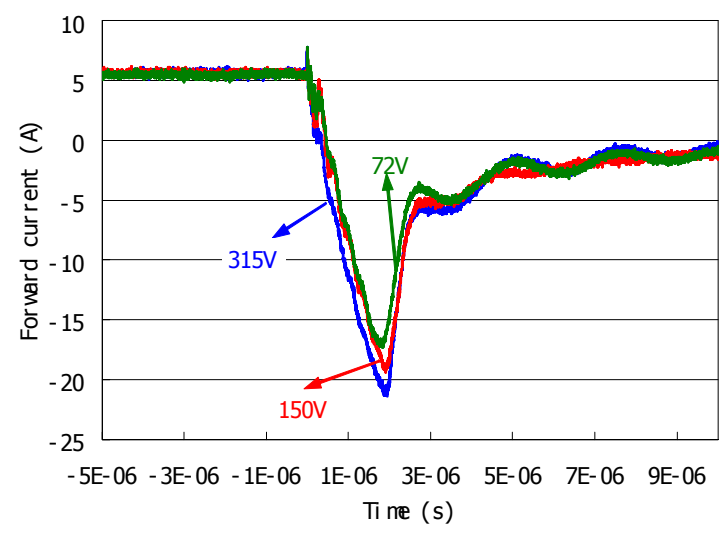

Fig. 7. Reverse recovery waveforms of the Si diode at $I_{F}=6 \mathrm{~A}$ (IR 20ETS08).

In addition, it should be noted that $D_{P}$ is a function of temperature and mobility of holes:

$$
D_{p}=\frac{k T}{q} \mu_{p}
$$

$\mu_{p}$ is also affected by temperature. From [16], for silicon, it can be determined by

$$
\mu_{p}=495\left(\frac{T}{300}\right)^{-2.2} \text {. }
$$

The final effect is that $D_{P}$ decreases and $Q_{I}$ increases as temperature increases. Consequently, the power loss during the reverse recovery of $\mathrm{Si}$ diodes increases with temperature, which is another reason for the large switching power losses in $\mathrm{Si}$ diodes.

\section{TESTS OF Si/SiC HYBRID INVERTER}

As discussed above, SiC Schottky diodes have smaller onstate resistance, and better reverse recovery behaviors, which greatly reduce the power losses in themselves. More importantly, if SiC Schottky diodes work as anti-parallel diodes, the power loss in the switches due to the reverse recovery current in diodes will be also reduced. Therefore, inverter applications are one of the potential areas for $\mathrm{SiC}$ Schottky diodes.

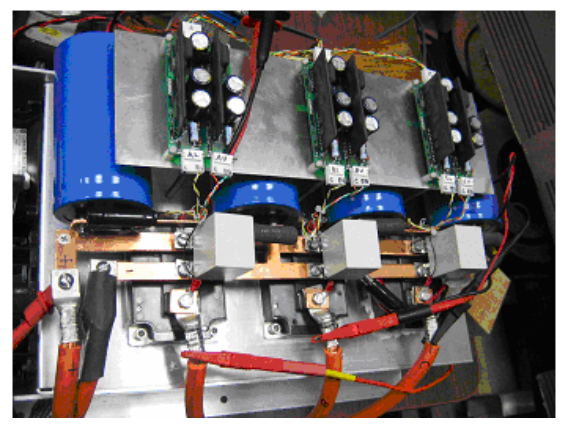

Fig. 8. Si/SiC Hybrid Inverter

A hybrid inverter was built using the hybrid $1200 \mathrm{~V} / 300 \mathrm{~A}$ IGBT Module (Si IGBT and SiC Schottky diode from Cree) as shown in Fig. 8 [19]. It was tested with an R-L load at different power levels and switching frequencies in order to study efficiencies of the inverter at various operating points. Fig. 9 shows inverter efficiency versus its output power for three switching frequencies at the same fundamental output frequency of $50 \mathrm{~Hz}$. As expected, the test results demonstrate that the inverter efficiencies increase with higher output power from $0.5 \mathrm{~kW}$ to $8.3 \mathrm{~kW}$, and decreases with higher switching frequency; specifically, from $5 \mathrm{kHz}$ to $10 \mathrm{kHz}$, it deceased by $1.78 \%$, and $3.78 \%$ from $10 \mathrm{kHz}$ to $15 \mathrm{kHz}$.

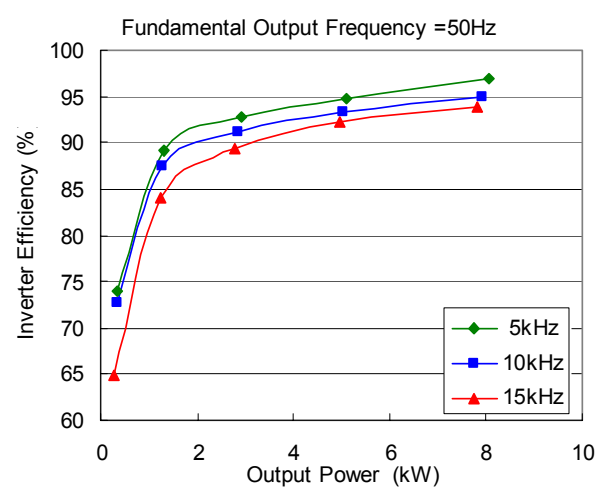

Fig. 9. Inverter efficiency vs. output power at different switching frequencies.

The hybrid module is in the same package with Powerex 1200V/300A Si IGBT/diode Module (CM300DY-24NF). The same tests were done for the Si inverter built using the Si IGBT/diode modules. Figs. 10-11 compare the efficiency of these two inverters at different switching frequencies. The average power loss reduction in the hybrid inverter is between $11.5 \%-19.4 \%$ of the total power loss of the $\mathrm{Si}$ inverter.

The efficiency of the hybrid inverter can be estimated by using the models presented previously in this paper (for 


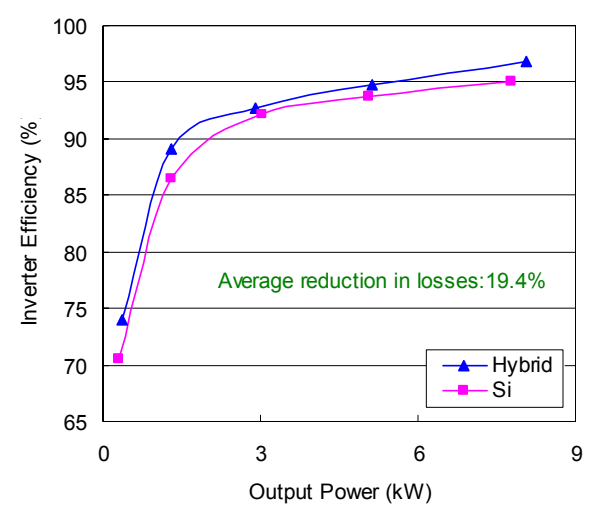

Fig. 10. Efficiency of the hybrid inverter and the Si inverter. (Switching frequency $=5 \mathrm{kHz}$ ).

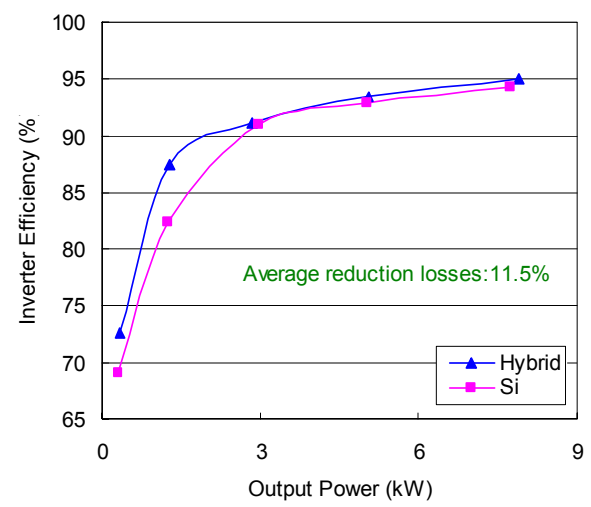

Fig. 11. Efficiency of the hybrid inverter and the Si inverter. (Switching frequency $=10 \mathrm{kHz}$ )

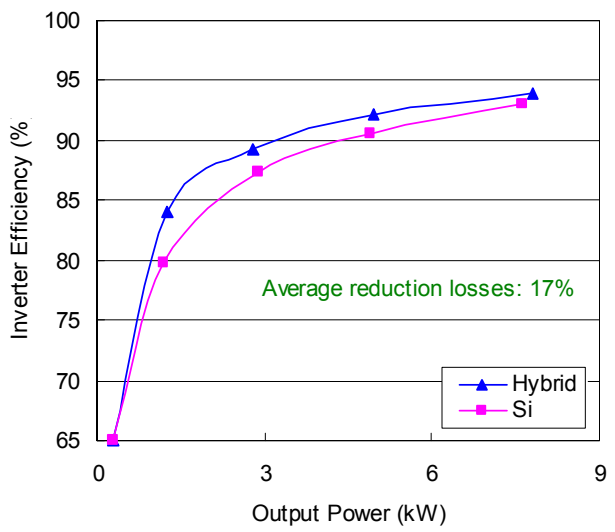

Fig. 12. Efficiency of the hybrid inverter and the Si inverter. (Switching frequency $=15 \mathrm{kHz}$ )

IGBT models, refer to papers [17] [18], and the parameters used in the simulation are also from tests and datasheet). In Figs. 13-15, the solid lines are the efficiency obtained from simulations (for fundamental output frequency of $50 \mathrm{~Hz}, \mathrm{DC}$ voltage of $315 \mathrm{~V}$ ), and scatter points are from test results. The simulation results fit the test results very well for most of the test points, and only deviate at the low current level (compared to the device rating 300A). Thus, the models presented in section II are effective in inverter applications.

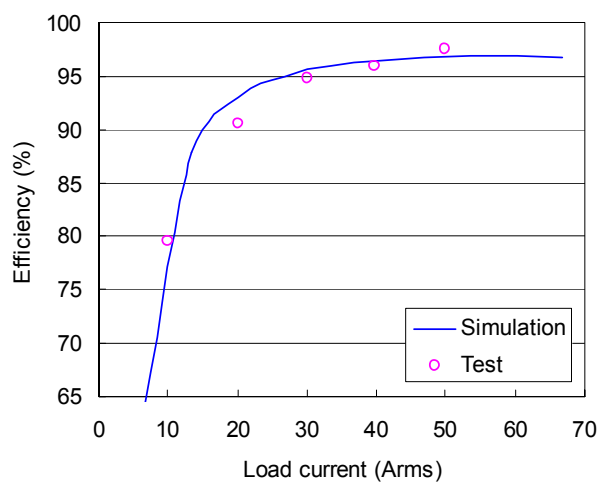

Fig. 13. Efficiency of the hybrid inverter vs. load current. (Fundamental output frequency $=50 \mathrm{~Hz}$, Switching frequency $=5 \mathrm{kHz}$ )

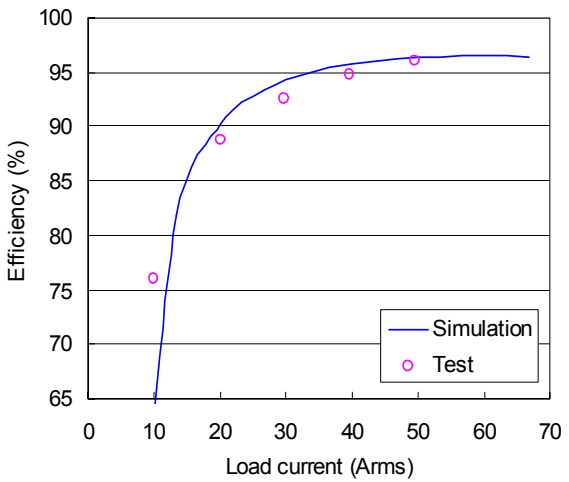

Fig. 14. Efficiency of the hybrid inverter vs. load current. (Fundamental output frequency $=50 \mathrm{~Hz}$, Switching frequency $=10 \mathrm{kHz}$ )

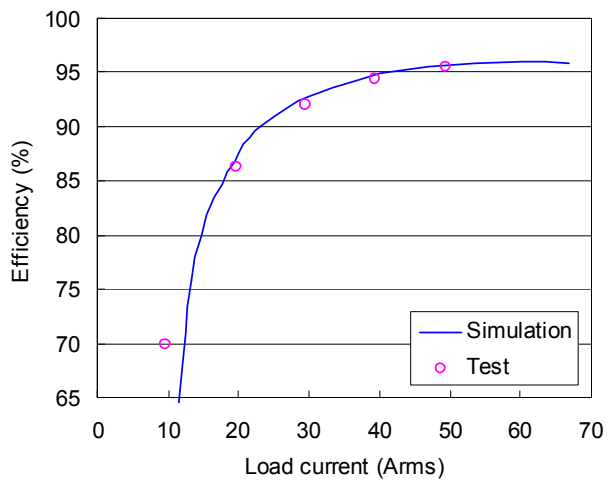

Fig. 15. Efficiency of the hybrid inverter vs. load current. (Fundamental output frequency $=50 \mathrm{~Hz}$, Switching frequency $=15 \mathrm{kHz}$ )

\section{CONCLUSION}

The models presented in this paper are specialized for system-level simulations. These models not only described static and dynamic characteristics of SiC Schottky power diodes, but also reflect their dependence on temperature. Thus, they are very useful and effective to estimate the power losses of SiC Schottky diodes and to predict device temperatures. The models were also used to estimate the efficiency of a Si IGBT/SiC Schottky diode hybrid inverter. 
A good match between simulation results and test results is shown.

In addition, according to the dynamic models presented in this paper, the power loss of $\mathrm{Si}$ diodes during turn off increases quickly as forward current, reverse voltage, and temperature go up, while that of SiC Schottky diodes only changes with reverse voltage. Thus, SiC Schottky diodes are better than Si diodes as anti-paralleled diodes, especially for high power and high temperature applications. This is accordant to the tests results of the hybrid inverter and the $\mathrm{Si}$ inverter in this work.

\section{ACKNOWLEDGMENT}

The authors would like to thank Charles Scozzie of Army Research Lab for funding the program that made the $\mathrm{Si} / \mathrm{SiC}$ Hybrid IGBT modules and Anant Agarwal of Cree Inc. for supplying these modules. The authors would also like to thank Jeremy Campbell of ORNL for his assistance with the experimental testing of the inverters.

\section{REFERENCES}

[1] K. J. Tseng, S. Pan, C. F. Foo, "Issues in power diode modeling for circuit simulation," Proceedings of 1997 International Conference on Power Electronics and Drive Systems, vol. 1, 26-29 May 1997, pp. 7884.

[2] D. Linzen, R. W. D. Doncker, "Simulation of power losses with MATLAB/Simulink using advanced power device models," Proceeding of 2002 IEEE Workshop on Computers in Power electronics, 3-4 June 2002, pp. 71-75.

[3] B. J. Baliga, Modern Power Devices, John Wiley \& Sons Inc., New York, 1987.

[4] F. Roccaforte, S. Libertino, F. Giannazzo, C. Bongiorno, F. L. Via, and V. Raineri, "Ion irradiation of inhomogeneous Schottky barriers on silicon carbide," Journal of Applied Physics, vol. 97, issue 12, 15 June 2005.
[5] S. Selberherr, Analysis and Simulation of Semiconductor Devices, Springer-Verlag/Wien, New York, 1984.

[6] S. Kagamihara, H. Matsuura, "Parameters required to simulate electric characteristics of $\mathrm{SiC}$ devices for $n$-type 4H-SiC," Journal of Applied Physics, vol. 96, no.10, 15 Nov. 2004, pp. 5601-5606.

[7] M. Roschke, F. Schwierz, "Electron mobility models for $4 \mathrm{H}, 6 \mathrm{H}$, and 3C SiC," IEEE Transactions on Electron Devices, vol. 48, no. 7, July 2001, pp. 1442-1447.

[8] M. Ruff, H. Mitlehner, R. Heibig, "SiC devices: physics and numerical simulation," IEEE Transactions on Electron Devices, vol. 41, no.6, June 1994, pp.1040-1054.

[9] F. Roccaforte, F. L. Via, A. L. Magna, S. D. Franco, and V. Raineri, "Silicon carbide pinch rectifiers using a dual metal Ti-Ni2Si Schottky barrier," IEEE Transactions on Electron Devices, vol. 50, no. 8, August 2003, pp.1741-1747.

[10] Datasheet of silicon carbide Schottky diode-CSD10120, www.cree.co $\mathrm{m} /$ products/pdf/CSD10120.pdf

[11] Datasheet of silicon carbide Schottky diode-CSD05120, www.cree.co $\mathrm{m} /$ products/pdf/CSD05120.pdf

[12] Datasheet of silicon carbide Schottky diode-CSD10060, www.cree.co $\mathrm{m} /$ products/pdf/CSD10060.pdf

[13] N. Mohan, T. M. Undeland, W. P. Robbins, Power Electronics, $2^{\text {nd }}$ Edition, John Wiley \& Sons Inc., 1995, pp.516-517.

[14] S. Hodge, "SiC power Schottky diodes in power factor correction circuits," Cree Application Note.

[15] J. R. Collard, F. Sterzer, "Current dependence of the depletion-layer capacitance of reverse-biased P-N junctions," Applied Physics Letters, vol. 5, no. 8, October 1964, pp. $165-167$.

[16] B. J. Baliga, Power Semiconductor Devices, PWS Publishing Company, Boston, 1996, pp. 11.

[17] H. Zhang, L. M. Tolbert, B. Ozpineci, M. Chinthavali, "A SiC-based converter as a utility interface for a battery system," IEEE Industry Applications Society Annual Meeting,, October 8-12, 2006, Tampa, Florida.

[18] H. Zhang, L. M. Tolbert, B. Ozpineci, M. Chinthavali, "Power losses and thermal modeling of $4 \mathrm{H}-\mathrm{SiC}$ VJFET inverter," IEEE Industry Applications Society Annual Meeting, October 2-6, 2005, Hong Kong, China, pp. 2630-2634.

[19] B. Ozpineci, M. Chinthavali, A. Kashyap, L. M. Tolbert, A. Mantooth, "A $55 \mathrm{~kW}$ three-phase inverter with Si IGBTs and SiC Schottky diodes," IEEE Applied Power Electronics Conference, March 19-23, 2006, Dallas, Texas, pp. 448-454. 